\title{
Comparison of ontology alignment systems across single matching task via the McNemar's test
}

\author{
MAJID MOHAMMADI, Delft University of Technology \\ AMIR AHOOYE ATASHIN, Ferdowsi University of Mashhad \\ WOUT HOFMAN, TNO Research Institute \\ YAOHUA TAN, Delft University of Technology
}

\begin{abstract}
Ontology alignment is widely-used to find the correspondences between different ontologies in diverse fields. After discovering the alignments, several performance scores are available to evaluate them. The scores typically require the identified alignment and a reference containing the underlying actual correspondences of the given ontologies. The current trend in the alignment evaluation is to put forward a new score (e.g., precision, weighted precision, semantic precision, etc.) and to compare various alignments by juxtaposing the obtained scores. However, it is substantially provocative to select one measure among others for comparison. On top of that, claiming if one system has a better performance than one another cannot be substantiated solely by comparing two scalars. In this paper, we propose the statistical procedures which enable us to theoretically favor one system over one another. The McNemar's test is the statistical means by which the comparison of two ontology alignment systems over one matching task is drawn. The test applies to a $2 \times 2$ contingency table which can be constructed in two different ways based on the alignments, each of which has their own merits/pitfalls. The ways of the contingency table construction and various apposite statistics from the McNemar's test are elaborated in minute detail. In the case of having more than two alignment systems for comparison, the family-wise error rate is expected to happen. Thus, the ways of preventing such an error are also discussed. A directed graph visualizes the outcome of the McNemar's test in the presence of multiple alignment systems. From this graph, it is readily understood if one system is better than one another or if their differences are imperceptible. The proposed statistical methodologies are applied to the systems participated in the OAEI 2016 anatomy track, and also compares several well-known similarity metrics for the same matching problem.
\end{abstract}

Additional Key Words and Phrases: ontology alignment; McNemar’s test; family-wise error rate; anatomy; OAEI;

ACM Reference format:

Majid Mohammadi, Amir Ahooye Atashin, Wout Hofman, and Yaohua Tan. 2017. Comparison of ontology alignment systems across single matching task via the McNemar's test. ACM Trans. Knowl. Discov. Data. 0, 0, Article 0 ( 2017 ), 17 pages.

DOI: 0000001.0000001

\section{INTRODUCTION}

With the advancement in information technology, data these days come from various sources. Such data have multiple salient but unwelcome features: they are big, dynamic and heterogeneous. There are solutions to cope with any of these features, and ontology alignment (or mapping/matching) is a remedy to data heterogeneity (Euzenat et al. 2007).

Given the source and target ontologies for alignment, a correspondence is defined as the mapping of one concept in the source to one concept in the target ontology. For discovering correspondences, it is typical

ACM acknowledges that this contribution was authored or co-authored by an employee, or contractor of the national government. As such, the Government retains a nonexclusive, royalty-free right to publish or reproduce this article, or to allow others to do so, for Government purposes only. Permission to make digital or hard copies for personal or classroom use is granted. Copies must bear this notice and the full citation on the first page. Copyrights for components of this work owned by others than ACM must be honored. To copy otherwise, distribute, republish, or post, requires prior specific permission and/or a fee. Request permissions from permissions@acm.org.

(c) 2017 ACM. 1556-4681/2017/0-ART0 $\$ 15.00$

DOI: 0000001.0000001

ACM Transactions on Knowledge Discovery from Data, Vol. 0, No. 0, Article 0. Publication date: 2017. 
to utilize one or more similarity measures. There are three different categories for the similarity calculation (Euzenat et al. 2007). The first category is the string-based measures which only considers the text of concepts to compute their similarities (Cohen et al. 2003; Levenshtein 1966; Stoilos et al. 2005). Another group is the linguistic-based similarity measures which consider the linguistic relations, e.g. synonym, antonym, hypernym, etc., between the strings of two concepts. The linguistic-based similarity measures usually take advantages of WordNet (Miller 1995) to discover the similarity. The third class is the structural-based measures which take into account the position of concepts in their ontologies.

Yet another approach is to match the entities of two given ontologies based on their instances (Xue and Wang 2015). The underlying assumption behind this type of alignment is that two entities are similar provided that they share, more or less, analogous instances.

Traditionally, the challenge of ontology alignment was to come up with a new similarity measure and then to find the interrelation between the ontologies (Stoilos et al. 2005). However, this focus has moved to take advantages of various similarity measures and try to reason correspondences based on the outcomes of various metrics (Jan et al. 2012; Nagy et al. 2006).

An alignment, which is the result of any standard ontology matching system, comprises a set of correspondences, mapping various concepts of one ontology to those of the other. It is the common practice to find the goodness of an alignment system by comparing its output with the actual reference alignment which is in hand. The typical performance scores are the precision and recall along with their variation such as relaxed precision and recall (Ehrig and Sure 2004), semantic precision and recall (Euzenat 2007), and so on. However, it is controversial to select the appropriate performance score in different cases. For instance, the comparison based on precision and recall would lead to totally different results. A system can be quite precise and discover as few false correspondences as possible, e.g., high precision, but could be conservative and not be able to detect an acceptable portion of correspondences, e.g., low recall. In addition to the selection of a performance metric, claiming the superiority of a system against one another cannot be substantiated merely by comparing the acquired scores. The difference between the performance measures of two systems could be small and imperceptible, thereby asserting the superiority of one system might not be correct. One approach to support such allegations and verify if the difference between two systems is substantial would be the statistical analysis. In this article, the appropriate procedures are put forward to statistically opt for one system if it has an enhanced performance than the other.

A note of caution is in order at this point, however. According to the no free lunch theorem (Wolpert 2012; Wolpert and Macready 1997), there is no context-independent reason to favor one strategy (or optimization method) over one another, and the average performances of all strategies over all possible problems are the same. It is drawn, as a result, that the superior performance of one method over one another is due to its better fitness to the nature of the problem, not because of its inherent features. Any claim of performing the best in a general sense must be questioned and faced with doubts.

The no free lunch theorem is firstly introduced in the supervised machine learning realm (Wolpert 1996), but it is generalized to any optimization problem afterward (Wolpert and Macready 1997). Therefore, the results of the no free lunch theorem are also correct for the ontology matching problem, and the preferred alignment can be only recognized in one particular context.

To date, the attempt of claiming if one alignment system is better than one another has been solely concentrated on employing a new performance score, e.g. semantic precision, relaxed precision, etc. (Ehrig and Sure 2004; Euzenat 2007; Ritze et al. 2013). If there are multiple pairs of ontologies for comparison, the superiority of a system is dedicated only if its average performance across multiple pairs of ontologies is higher than the rest. Statistically speaking, the average performance is unsafe and inappropriate: it is highly sensitive to outliers and having higher average performance does not necessarily indicate the superiority since the difference might be 
imperceptible and insignificant (Demšar 2006). In the case of existing only one pair of ontologies, on the other hand, the comparison is merely performed by the juxtaposition of the performance metric of various systems.

As a complement to the no free lunch theorem, this article aims to consider the statistical hypothesis testing to find the best ontology alignment on a particular task. Employing the appropriate statistical test, one can determine if one alignment system outperforms one another with substantial statistical evidence. Instead of comparing one alignment with the reference one, the recommended methodology here takes the reference along with two alignments under comparison as the inputs and states if one of them statistically outperforms the other. Thus, the expected outcome is not a score but the statement of superiority of an alignment in comparison with one another.

In the case that there are multiple tasks, various statistics such as Wilcoxon signed-rank and Friedman tests can be applied to a particular performance score obtained for each matching task (Mohammadi et al. 2018). In order words, the performance scores obtained from each task become the samples, hence the difference between systems can be gauged by conducting statistical tests over the samples. However, it is not the case for comparison over one matching task since there is no such samples.

The McNemar's test is the statistical means by which the various matching systems can be compared over one matching task. This test can be applied to the paired nominal data summarized in a contingency table with a dichotomous trait. Interestingly, the outcome of two alignment systems can be viewed as dichotomous (i.e., correct and incorrect correspondences) of two experiments (i.e., two alignment systems). Therefore, the McNemar's test suits for comparison of alignments. However, Summing up the results of alignments in a contingency table would be challenging and might erupt discussions. We present two ways to build such a contingency table whose applicabilities is conceptually similar to those of recall and F-measure. Further, four statistics from the McNemar's tests are considered, and their advantages and pitfalls are discussed. In the case of having two systems for comparison, the McNemar's test can be simply applied. If more than two alignments are available, all pairwise comparisons must be performed. In this case, the family-wise error rate (FWER) is likely to happen and must be controlled (Shaffer 1995). The appropriate procedures for the FWER prevention are elaborated as well.

We leverage the proposed methodology across the systems participated in the OAEI 2016 anatomy track, and the corresponding results are visualized by a directed graph. This graph indicates if the difference between each pair of systems are significant or not. Our investigation shows that AML and CroMatcher are the top two systems while DKP-AOM and Alin are the ones with reduced accomplishment. We further compare the stringbased similarity measures over this track because many correspondences can be easily discovered by comparing the strings. The N-gram and Levenstein distances are the ones with the maximum discovery with respect to others.

The contribution of this paper can be summarized as

- The utilization of the McNemar's test to conduct the comparison of alignment systems.

- Two ways of using the McNemar's test is proposed which are conceptually identical to those of recall and F-measure.

- The technique for the family-wise error rate is thoroughly discussed.

- The outcome of the statistical procedure for comparison of multiple systems is visualized by a directed graph.

- The systems participated in the OAEI anatomy track are compared and the corresponding results are reported.

This article is structured as follows. The ways of the contingency table construction are expounded in Section II, and the appropriate statistics from the McNemar's test are discussed in Section III. The family-wise error rate 
and the ways of adjusting the p-values are studied in Section IV. Section V dedicates to the experiments of the statistical procedures over the anatomy track, and the paper is concluded in Section VI.

\section{CONTINGENCY TABLE CONSTRUCTION}

The McNemar's test is applicable when there are two experiments over $\mathrm{N}$ samples (McNemar 1947). Let the outcome of each test be either positive or negative; then, a simple contingency table would be as Table 1 .

Table 1. A simple contingency table

\begin{tabular}{ccccc}
\hline & \multicolumn{4}{c}{ Exp. 2 } \\
& & - & + & sum \\
\hline Exp. 1 & - & $n_{00}$ & $n_{01}$ & $n_{0 .}$ \\
& + & $n_{10}$ & $n_{11}$ & $n_{1 .}$ \\
& sum & $n_{.0}$ & $n_{.1}$ & $\mathrm{~N}$ \\
\hline
\end{tabular}

In this table, $n_{00}$ and $n_{11}$ are called the accordant pair and are respectively the number of times both experiments produce positive and negative outcomes. The discordant pair, i.e. $n_{01}$ and $n_{10}$, are the number of times the results of experiments are in contradiction; $n_{01}$ is the number of experiments which the first outcome is negative while the second one is positive and $n_{10}$ is the other way around.

In the ontology matching case, the positive or negative outcome can be defined in two ways, each of which has its own merits and is suitable for particular situations.

For two given ontologies, let $R$ be the reference alignment containing a set of correct correspondences and $A_{1}$ and $A_{2}$ be two alignments retrieved by two different systems. In the first approach of the contingency table construction, the focus is solely on the truly discovered alignments, thereby ignoring the concepts which have not correctly mapped. Hence, $n_{00}$ and $n_{11}$ are respectively the number of false correspondences and the number of correct correspondences jointly identified by both systems. $n_{01}$ (and similarly $n_{10}$ ) is the number of correspondences correctly discovered by $A_{2}$, but not by $A_{1}$. These elements can be written as

$$
\left\{\begin{aligned}
n_{00} & =\left|R-\left(A_{1} \cup A_{2}\right)\right| \\
n_{01} & =\left|\left(A_{2} \cap R\right)-A_{1}\right| \\
n_{10} & =\left|\left(A_{1} \cap R\right)-A_{2}\right| \\
n_{11} & =\left|A_{1} \cap A_{2} \cap R\right|
\end{aligned}\right.
$$

where |.| indicates the cardinality operator. This approach is conceptually similar to recall as it does not consider the wrong correspondences in the alignments. We again accent that the approach of this article is distinct from the performance measures, including recall, as we compare two alignments and do not produce any score indicating the fineness of a system.

An example elaborates the issue of this approach. Assume that two systems could discover the complete reference alignment, i.e. $A_{1}=A_{2}=R$. In this case, $n_{01}=n_{10}=0$ which means that they are equally well (it is discussed in further sections that $n_{01}$ and $n_{10}$ are the only important pair for the McNemar's test). Now, suppose that $A_{1}=R$ and $A_{2}=R+B$, where $B$ is a set of correspondences which are not in $\mathrm{R}$ (falsely discovered by $A_{2}$ ). In this case, $n_{01}$ is the same as $n_{10}$ which again indicates that their performances are indiscernible. However, it is plain to grasp that $A_{1}$ is more reliable as it does not mistakenly discover any correspondences. Statistically speaking, this approach does not take into account the false positive and only considers the true positive. Nonetheless, such an approach is suitable for occasions where the goal is to have as many correspondences as possible so that the false discovery does not have a profound impact. 
The second approach of building the contingency table avoids the foregoing pitfall and consider the false discovery as well. Since it considers the truly unmapped pairs of concepts, obtaining the elements of the contingency table is of higher complexity in comparison with the previous approach. Therefore, it is necessary to explain how to obtain each element of the table individually.

$n_{00}$ is the number of correspondences which are wrongly discovered by both alignments. Hence it includes the correspondences which are in $\mathrm{R}$ but not in $A_{1}$ or $A_{2}$ plus the correspondences which are in both $A_{1}$ and $A_{2}$ but not in R, i.e. $n_{00}=\left|R-\left(A_{1} \cup A_{2}\right)\right|+\left|\left(A_{1} \cap A_{2}\right)-R\right| . n_{10}$ is the number of truly discovered correspondences by $A_{1}$ which are not in $A_{2}$ plus the correspondences which are falsely identified only by $A_{2}$ and not by $A_{1}$, i.e. $n_{10}=\left|\left(A_{1} \cap R\right)-A_{2}\right|+\left|A_{2}-A_{1}-R\right|$. By the same token, $n_{01}$ can also be obtained. $n_{11}$ is a bit more challenging as the total number of possible correspondences between two ontologies is required. Let this number be $T$, one possibility for $T$ is to multiply the number of concepts of two ontologies, i.e. $T=n \times m$ where $n$ and $m$ are the numbers of candidate concepts for matching in two ontologies. Thus, $n_{11}=\left|A_{1} \cap A_{2} \cap R\right|+\left|(T-R)-\left(A_{1} \cup A_{2}\right)\right|$. The statistics considered in this paper only need the discordant pair; therefore the value of $n_{11}$ and subsequently $T$ is not taken into account. The elements as mentioned earlier of the contingency table from the second approach can be summarized as:

$$
\left\{\begin{array}{l}
n_{00}=\left|R-\left(A_{1} \cup A_{2}\right)\right|+\left|\left(A_{1} \cap A_{2}\right)-R\right| \\
n_{01}=\left|\left(A_{2} \cap R\right)-A_{1}\right|+\left|A_{1}-A_{2}-R\right| \\
n_{10}=\left|\left(A_{1} \cap R\right)-A_{2}\right|+\left|A_{2}-A_{1}-R\right| \\
n_{11}=\left|A_{1} \cap A_{2} \cap R\right|+\left|(T-R)-\left(A_{1} \cup A_{2}\right)\right|
\end{array}\right.
$$

This way of the contingency table construction considers the false correspondences as well. The foregoing example illustrates the advantages of these formulas. As $A_{1}=R$ and $A_{2}=R+B, n_{01}=0$ and $n_{10}=|B|$. The null hypothesis is thus rejected for large enough of $\mathrm{B}$, and $\mathrm{A}$ is claimed to be superior. Therefore, the false positive of $\mathrm{B}$ resulted in declaring $\mathrm{A}$ to be the better system. Note that this calculation is relative to the other system. In other words, it does not consider all the incorrectly identified correspondences, but the false correspondences are computed as the ones which are not in the rival system. As the goal is to compare two alignments together, it is entirely logical to find the relative false positive. This approach can be figuratively viewed as similar to F-measure due to its consideration of both true and false discoveries.

\section{MCNEMAR'S TEST}

The McNemar's test is applied to the contingency table constructed in the previous section. But before looking into the test, we digress briefly to explain the null hypothesis testing.

To leverage any statistical test, the null and alternative hypotheses are required. The null hypothesis $H_{0}$ states that the difference between two populations is insignificant, and the existing discrepancy is due to the sampling or experimental errors (Sheskin 2003). The alternative hypothesis, on the other hand, states the contrary: the difference between two populations is significant and not random.

To reject or retain $H_{0}$, we need to compute the p-value and compare it with significant level $\alpha$ which must be determined before running the test. The p-value is the probability of obtaining a result equal to, or even more extreme than, the observations given the null hypothesis is true (Sheskin 2003). If the p-value is less than the nominal significant level $\alpha$, then the null hypothesis is rejected, and it is drawn that the disparity between populations is significant.

In comparison of ontology alignment systems, the populations mentioned above are the outcomes of two systems. Therefore, the null hypothesis is that the difference between the outcomes of alignments is random and insignificant. The null hypothesis in the McNemar's test states that the two marginal probabilities of the 
contingency table are the same, i.e.

$$
\begin{aligned}
& p\left(n_{00}\right)+p\left(n_{01}\right)=p\left(n_{00}\right)+p\left(n_{10}\right) \\
& p\left(n_{10}\right)+p\left(n_{11}\right)=p\left(n_{01}\right)+p\left(n_{11}\right)
\end{aligned}
$$

where $p(a)$ indicates the probability of occurring the cell of Table 1 with the label $a$. After canceling out the $p\left(n_{00}\right)$ and $p\left(n_{11}\right)$ from the foregoing equations, the null and alternative hypotheses become

$$
\begin{array}{ll}
H_{0}: & p\left(n_{01}\right)=p\left(n_{10}\right) \\
H_{a}: & p\left(n_{01}\right) \neq p\left(n_{10}\right) .
\end{array}
$$

To compute the p-value of the null hypothesis (4), we consider four statistics from the McNemar's test and discuss their advantages and pitfalls in the hypothesis testing. The statistics studied here only work with the accordant pair of the contingency table. However, there is also an exact unconditional McNemar's test which takes into account the discordant pair of the contingency table (Suissa and Shuster 1991). The exact unconditional test is way more intricate than the McNemar's tests put forward here, but its power is approximately the same as other tests (Fagerland et al. 2013). Therefore, this test is ignored in this paper.

\subsection{The McNemar's asymptotic test}

The McNemar's asymptotic test assumes that $n_{01}$ is binomially distributed with $p=0.5$ and parameters $n=$ $n_{01}+n_{10}$ under the null hypothesis (McNemar 1947). The McNemar's asymptotic statistic

$$
\chi^{2}=\frac{\left(n_{01}-n_{10}\right)^{2}}{n_{01}+n_{10}}
$$

is distributed according to $\chi^{2}$ with one degree of freedom. This test is undefined for $n_{01}=n_{10}=0$.

To reject the null hypothesis, this test requires a sufficient number of data $\left(n_{01}+n_{10} \geq 25\right)$ since it might violate the nominal significant level $\alpha$ for the small sample size.

\subsection{The McNemar's exact test}

It is traditionally advised to use the McNemar's exact test when a small sample size is available in order not to exceed the nominal significant level. In this test, $n_{01}$ is compared to a binomial distribution with parameter $n=n_{01}+n_{10}$ and $p=0.5$. Thus, the $\mathrm{p}$-value for this test is obtained as

$$
\text { exact-p-value }=\sum_{x=n_{01}}^{n}\left(\begin{array}{l}
n \\
x
\end{array}\right)\left(\frac{1}{2}\right)^{2}
$$

The two-sided p-value is calculated by multiplication of the one-sided p-value by two. This test guarantees to have type I error rate below the nominal significant level $\alpha$.

\subsection{The McNemar's asymptotic test with continuity correction}

The main drawback of the McNemar's exact test, though preserving the nominal significant level, is conservatism: it unnecessarily generates large p-values so that the null hypothesis cannot be rejected. As a remedy to conservatism, Edwards (Edwards 1948) approximated the exact p-value by the following continuity corrected statistic

$$
\chi^{2}=\frac{\left(\left|n_{01}-n_{10}\right|-1\right)^{2}}{n_{01}+n_{10}}
$$

which is $\chi^{2}$-distributed with one degree of freedom. This test is also undefined for $n_{01}=n_{10}=0$. 


\subsection{The McNemar's mid-p test}

The continuity corrected method is not as conservative as the exact test, but it does not guarantee to preserve the nominal significant level. The mid-p approach propounds a way to trade off between the conservatism of the exact tests and the significant level transgression of the continuity correction approach (Lancaster 1961). To obtain the mid-p-value, a simple modification is required: the mid-p-value equals the exact p-value minus half the point probability of the observed test statistic (Fagerland et al. 2013). Hence, the p-value could be computed as

$$
\text { mid-p-value }=2 \text {-sided exact } \mathrm{p} \text {-value }-\left(\begin{array}{c}
n \\
n_{01}
\end{array}\right) 0.5^{n} \text {. }
$$

The McNemar's mid-p test resolves the conservatism of the exact test, but it does not guarantee theoretically to preserve the nominal significant level. In a recent study, however, it is investigated that the mid-p test has low type I error and does not violate the significant level. The continuity-corrected test, in contrast, indicated a high type I error, coming from the nature of asymptotic tests, as well as high type II error, inherited from the exact test. Thus, it is rational not to use the continuity-corrected test for the alignment comparison.

\section{FAMILY-WISE ERROR RATE AND P-VALUE ADJUSTMENT}

When there are two systems for comparison, the null hypothesis will be rejected if the obtained p-value is below the nominal significant level $\alpha$. If more than two alignments are available for comparison, the well-known family-wise error rate (FWER) might occur. FWER refers to the increase in the probability of type I error which is likely to violate the nominal significant level $\alpha$ when multiple populations are to be compared. To explain what FWER is, assume that there are 5 systems for comparison and the significant level is $\alpha=0.05$. If it is desired to do all the pairwise comparisons, then there are $k=5 \times 4 / 2=10$ hypotheses overall. For each of null hypotheses, the probability of rejection without occurring the type I error is $1-\alpha=0.95$. For all comparisons, on the other hand, the probability of not having any type I error in all the hypotheses is $(0.95)^{10}=0.6$. As a result, the probability of occurring at least one type I error increases to $1-0.6=0.4$, which is way higher than the nominal $\alpha=0.05$. This phenomenon is the so-called family-wise error rate.

To prevent this error, there are two primary approaches. Akin to the preceding example, the first approach is applicable when all the pairwise comparisons are desired. Conducting all pairwise comparisons are suitable when a comparison study of the existing systems in the literature or their competition in a competition like OAEI is desired. Another approach to control FWER is convenient when a new alignment system is proposed and it is to be compared with other existing ones. In the interest of simplicity, the former approach is called $N \times N$ comparisons and the latter is called $N \times 1$ comparisons.

\subsection{Controlling FWER in $N \times 1$ comparison}

When a new alignment system is proposed, it is usually compared with other existing ontology matchers. For comparing $n$ systems (including the proposed one) in this case, $k=n-1$ comparisons must be performed. There are four methods which can control the family-wise error rate in this case. These methods can be viewed as the p-value adjustment procedures which modify the p-values in a way that the adjusted p-values (APV) can be directly compared with the significance level while the nominal significant level is also preserved. Thus, a null hypothesis is rejected if its corresponding adjusted p-value is below the nominal $\alpha$.

Let $H_{i}, i=1, \ldots, k$ be all hypotheses for $n$ systems and $p_{i}, i=1, \ldots, k$ be their corresponding p-values. The Bonferroni's method (Dunn 1961) is the most straightforward way to prevent FWER. In this procedure, all the p-values are compared with the nominal significant level $\alpha$ divided by the total number of comparisons. In other words, the hypothesis $H_{i}$ is rejected if $p_{i}<\alpha / k$. Based on this equation, the adjusted p-value for the hypothesis $H_{i}$ is obtained by multiplying both sides of above inequality by $k$, i.e. $A P V_{i}=\min \left\{k \times p_{i}, 1\right\}$. Thus, $H_{i}$ is rejected 
if $A P V_{i}<\alpha$. This procedure, though simple, is too conservative: it retains the hypotheses which must be rejected by generating high APV.

In contrary to the single step Bonferroni's correction, there are step-up and step-down procedures which sequentially reject the null hypothesis. It is necessary to order p-values for sequential rejective procedures and we denote the ordered p-values as $p_{1} \leq p_{2} \leq \ldots \leq p_{k}$ and their corresponding hypotheses as $H_{1}, H_{2}, \ldots, H_{k}$.

The Holm's procedure (Holm 1979) is a step-down method which starts with the most significant (or the smallest) $\mathrm{p}$-value $p_{1}$. If $p_{1} \leq \frac{\alpha}{k}$, then $H_{1}$ is rejected, and $p_{2}$ is compared with $\frac{\alpha}{k-1}$. If $p_{2} \leq \frac{\alpha}{k-1}$, then $H_{2}$ is rejected, and $p_{3}$ is compared with $\frac{\alpha}{k-2}$. This procedure continues until a hypothesis is retained. In other words, each $p_{i}$ in the Holm's method is compared with $\frac{\alpha}{k+1-i}$ and it is rejected if it is below this value; otherwise, it is not rejected and the rest hypotheses are retained as well. The Holm's adjusted p-value is $A P V_{i}=\min \left\{v_{i}, 1\right\}$ where $v_{i}=\max \left\{(k-j) p_{j}: 1 \leq j \leq i\right\}$.

Similar to the Holm's procedure, the Holland's correction (Holland and Copenhaver 1987) is also a step-down method. Instead of comparing the p-values with $\frac{\alpha}{k+1-i}$, it compares each $p_{i}$ with $1-(1-\alpha)^{k-i}$. Thus, the adjusted $\mathrm{p}$-value is $A P V_{i}=\min \left\{v_{i}, 1\right\}$ where $v_{i}=\max \left\{1-\left(1-p_{j}\right)^{k+1-j}: 1 \leq j \leq i\right\}$. The Finner's procedure (Finner 1993) is almost the same as the Holland's technique and compares each $p_{i}$ with $1-(1-\alpha)^{\frac{k}{i}}$. The Finner's adjusted $\mathrm{p}$-value is $A P V_{i}=\min \left\{v_{i}, 1\right\}$ where $v_{i}=\max \left\{1-\left(1-p_{j}\right)^{\frac{k}{j}}: 1 \leq j \leq i\right\}$.

The Hochberg's method (Hochberg 1988) works in the opposite direction and starts with the largest p-value. It compares the largest $\mathrm{p}$-value with $\alpha$, the next largest with $\alpha / 2$ and it is terminated until a hypothesis is rejected. All the hypotheses with the smaller p-values are then rejected as well. The Hochberg's adjusted p-value is $A P V_{i}=\max \left\{(k-j) p_{j}:(k-1) \geq j \geq i\right\}$.

\subsection{Controlling FWER in $N \times N$ comparison}

For performing all the pairwise comparisons when $n$ systems are available, there are $k=n(n-1) / 2$ hypotheses overall. The Nemenyi's method (Nemenyi 1963) is exactly the Bonferroni's correction with $k$ is set to the $N \times N$ comparison, i.e. $k=n(n-1) / 2$. Thus, it has high type II error which results in not detecting the difference among the population when there is a de facto difference. The same modification of $k$ must be applied to other methods so that they are suitable for $N \times N$ comparison case.

There is also another sequential-rejective null hypothesis approach which is suitable for $N \times N$ comparison. This approach takes into account the logical relations between hypotheses. Shaffer (Shaffer 1986) discovered that the Holm's procedure could be improved when hypotheses are logically interrelated. In many scenarios, it is not feasible to get any combination of true and false hypotheses. In the pairwise comparison, for instance, it is not possible to have $\mu_{1}=\mu_{2}$ and $\mu_{2}=\mu_{3}$ but $\mu_{1} \neq \mu_{3}$. Thus, this case need not be protected against FWER.

Correction procedures which take into account the logical relations are similar to the Holm's correction: they start with the most significant (or the smallest) $\mathrm{p}$-value but compare it with $\alpha / t_{1}$, where $t_{1}$ is the maximum number of hypotheses which can be retained at the first step. If $p_{1}<\alpha / t_{1}$, then the corresponding hypothesis $H_{1}$ is rejected, and $p_{2}$ is compared with $\alpha / t_{2}$. If $H_{2}$ is rejected, then $p_{3}$ is compared with $\alpha / t_{3}$ and so on. The procedure terminates at the stage $j$ if $H_{j}$ cannot be rejected. The remaining hypotheses with bigger p-values than $p_{j}$ are also retained. The adjusted $\mathrm{p}$-value for the sequential corrective methods is $A P V_{i}=\min \left\{v_{i}, 1\right\}$ where $v_{i}=\min \left\{t_{i} \times p_{i}, 1\right\}$.

There are two well-known techniques which consider the logical relations of hypotheses: Shaffer's and Bergmann's. These methods differ in their way to obtain the maximum number of true hypotheses at each level. The Holm's procedure simply assigns the maximum number of true hypothesis at the stage $j$ to the number of remaining hypothesesat the $j^{t h}$ stage, i.e. $t_{j}=k-j+1$.

In the Shaffer's method (Shaffer 1986), the possible numbers for true hypothesis and consequently $t_{j}$ is obtained 
by the following recursive formula

$$
S(k)=\bigcup_{j=1}^{k}\left\{\left(\begin{array}{l}
2 \\
j
\end{array}\right)+x: x \in S(k-j)\right\}
$$

where $S(k)$ is the set of all possible numbers of true hypotheses when there are $k$ alignments for comparison and $S(0)=S(1)=0 . t_{j}$ is simply computed based on the set $S(k)$.

Similar to the Shaffer's method, the Bergmann's method (Bergmann and Hommel 1988) use the logical interrelations between the hypotheses but dynamically estimates the maximum number of true hypotheses at the stage $j$, given that $j-1$ hypotheses are rejected.

To do so, they defined the exhaustive which is an index set of hypotheses $I \subseteq\{1, \ldots, m\}$ where exactly all the hypotheses $H_{j}, j \in I$ can be true. For instance, let $A_{1}, A_{2}$, and $A_{3}$ be three alignments under study. If the null hypothesis between $A_{1}$ and $A_{2}$ is rejected, e.g., $A_{1} \neq A_{2}$, then it is not possible that both hypothesis $A_{1}=A_{3}$ and $A_{2}=A_{3}$ be correct because the performance of $A_{3}$ cannot be the same as $A_{1}$ and $A_{2}$ while $A_{1}$ and $A_{2}$ have been already declared significantly different.

Having calculated the exhaustive set, any hypothesis $H_{j}$ is rejected if $j \notin A$ where $A$ is the acceptance set which is retained and defined as

$$
A=\bigcup\left\{\text { I: I exhaustive, } \min \left\{P_{i}: i \in I\right\}>\alpha /|I|\right\}
$$

The Bergmann's method is one of the most powerful procedures when $N \times N$ comparison is demanded since it dynamically takes into account the logical relations of hypothesis. However, building the exhaustive set is time-consuming especially if more than nine systems are available for comparison.

\section{RESULTS}

In this section, the recommended statistical procedures are applied to the OAEI 2016 anatomy track, and the corresponding results are reported. Further, different string similarity metrics are compared and ranked according to the number of correct discoveries.

We have two ways of obtaining the contingency table, four McNemar's statistics and four ways to prevent FWER. Therefore, there are totally 32 states for comparison. On account of simplicity (and probably for the exclusion of duplication), we only consider four states: the two ways of building the contingency table compared with the McNemar's mid-p test and controlling FWER by the Nemenyi's and Bergmann's correction techniques, the most conservative and the most robust methods. The underlying reason behind the mid-p test selection is that it is not as conservative as the exact test and it is less likely to violate the nominal significant level $\alpha$ rather than the asymptotic test.

The anatomy track has been a part of OAEI since 2011 and its aim is to find the alignment between the Adult Mouse Anatomy and a part of the NCI Thesaurus related to the human anatomy. We select 10 systems participated in the OAEI 2016 for conducting the comparison: Alin (da Silva 2016), AML (Faria et al. 2013), CroMatcher (Achichi et al. 2016), DKP-AOM (Amrouch et al. 2016), FCA-Map (Zhao and Zhang 2016), Lily (Wang and Xu 2008), LogMapLite (Jiménez-Ruiz and Grau 2011), LPHOM (Megdiche et al. 2016), LYAM (Achichi et al. 2016), and XMap (Djeddi and Khadir 2010).

The contingency table is built by two foregoing methodologies. The values of $n_{01}$ and $n_{10}$ for the first and second way of table construction are arranged in Tables 2 and 3 , respectively. For the interest of simplicity, $n_{01}$ and $n_{10}$ are tabulated in one single table for each perspective (below and upper diagonal). To compare the $i^{t h}$ and $j^{t h}$ systems in each approach, $(i, j)$ and $(j, i)$ elements of this table are taken as $n_{01}$ and $n_{10}$, where $(i, j)$ is the element at the $i^{t h}$ row and $j^{t h}$ column. For instance, let's compare Alin and AML systems. In the first perspective, $n_{01}=911$ which means that there are 911 correspondences discovered by AML but not by Alin. And, $n_{10}=0$ indicates that there are no correspondences identified by Alin but not by AML. In the second perspective, on 
Table 2. The $n_{01}$ and $n_{10}$ for constructing the contingency table from the first point of view which does not consider the false positives(see Eq. (1)). For comparing the $i^{t h}$ and $j^{t h}$ systems, $n_{01}=(i, j)$ and $n_{10}=(j, i)$ where $(i, j)$ is the element at the $i^{\text {th }}$ row and the $j^{\text {th }}$ column in the table.

\begin{tabular}{|c|c|c|c|c|c|c|c|c|c|c|}
\hline & $: \Xi$ & $\sum_{\&}$ & 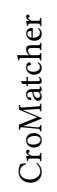 & 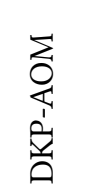 & 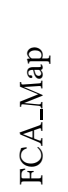 & $\stackrel{\lambda}{\rightrightarrows=}$ & 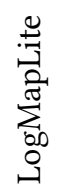 & 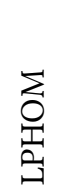 & 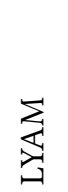 & $\sum_{x}^{\frac{\pi}{x}}$ \\
\hline Alin & 0 & 0 & 13 & 405 & 2 & 18 & 2 & 52 & 3 & 0 \\
\hline AML & 911 & 0 & 62 & 1214 & 184 & 237 & 328 & 339 & 118 & 134 \\
\hline CroMatcher & 873 & 11 & 0 & 1170 & 176 & 216 & 311 & 314 & 108 & 124 \\
\hline DKP-AOM & 102 & 0 & 7 & 0 & 0 & 13 & 0 & 49 & 1 & 0 \\
\hline FCA_Map & 763 & 34 & 77 & 1064 & 0 & 161 & 167 & 253 & 51 & 58 \\
\hline Lily & 713 & 21 & 51 & 1011 & 95 & 0 & 176 & 210 & 45 & 60 \\
\hline LogMapLite & 597 & 12 & 46 & 898 & 1 & 76 & 0 & 203 & 5 & 19 \\
\hline LPHOM & 646 & 22 & 48 & 946 & 86 & 109 & 202 & 0 & 43 & 39 \\
\hline LYAM & 823 & 27 & 68 & 1124 & 110 & 170 & 230 & 269 & 0 & 74 \\
\hline XMap & 804 & 27 & 68 & 1107 & 101 & 169 & 228 & 249 & 58 & 0 \\
\hline
\end{tabular}

Table 3. The $n_{01}$ and $n_{10}$ for constructing the contingency table from the second point of view which takes into account the false positives (see Eq. (2)). For comparing the $i^{t h}$ and $j^{t h}$ systems, $n_{01}=(i, j)$ and $n_{10}=(j, i)$ where $(i, j)$ is the element at the $i^{\text {th }}$ row and $j^{\text {th }}$ column in the table.

\begin{tabular}{|c|c|c|c|c|c|c|c|c|c|c|}
\hline & $\Xi$ & $\sum_{<}$ & 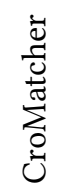 & 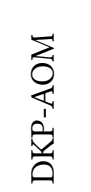 & 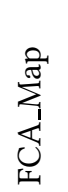 & $\stackrel{2}{ت}$ & 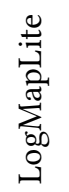 & 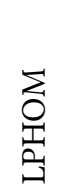 & 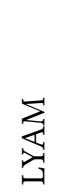 & $\sum_{x}^{\text {Jै }}$ \\
\hline Alin & 0 & 72 & 86 & 405 & 92 & 195 & 46 & 506 & 212 & 100 \\
\hline AML & 917 & 0 & 94 & 1214 & 252 & 396 & 368 & 777 & 298 & 203 \\
\hline CroMatcher & 879 & 42 & 0 & 1170 & 249 & 375 & 351 & 749 & 298 & 204 \\
\hline DKP-AOM & 108 & 72 & 80 & 0 & 90 & 190 & 50 & 509 & 210 & 100 \\
\hline FCA_Map & 769 & 84 & 133 & 1064 & 0 & 323 & 181 & 691 & 220 & 135 \\
\hline Lily & 719 & 75 & 106 & 1011 & 170 & 0 & 219 & 617 & 234 & 138 \\
\hline LogMapLite & 597 & 74 & 109 & 898 & 55 & 246 & 0 & 648 & 186 & 107 \\
\hline LPHOM & 647 & 73 & 97 & 947 & 155 & 234 & 238 & 0 & 214 & 105 \\
\hline LYAM & 829 & 70 & 122 & 1124 & 160 & 327 & 252 & 690 & 0 & 142 \\
\hline XMap & 810 & 68 & 121 & 1107 & 168 & 324 & 266 & 674 & 235 & 0 \\
\hline
\end{tabular}

the other hands, $n_{01}=917$ and $n_{10}=72$. Comparing with the previous view, $n_{10}$ changes from 0 to 72 which means that AML has discovered 72 wrong correspondences while Alin has not. The little increase in $n_{01}$ is due to the false discovery rate of Alin (6 correspondences) in comparison to AML. As a result, it is grasped that the false discovery rate of Alin is less than AML while the true discovery rate of AML is way higher than Alin. If the McNemar's test rejects the null hypothesis, AML is thus concluded to have a better performance than Alin due to its higher true discovery rate. The comparison of other systems can be conducted likewise which clarifies the difference between two perspectives. 
Fig. 1. Comparison of alignment systems by the McNemar's mid-p test with the Nemenyi's correction while the false positive is ignored. The edge $A \rightarrow B$ indicates that $\mathrm{A}$ outperforms $\mathrm{B}$.

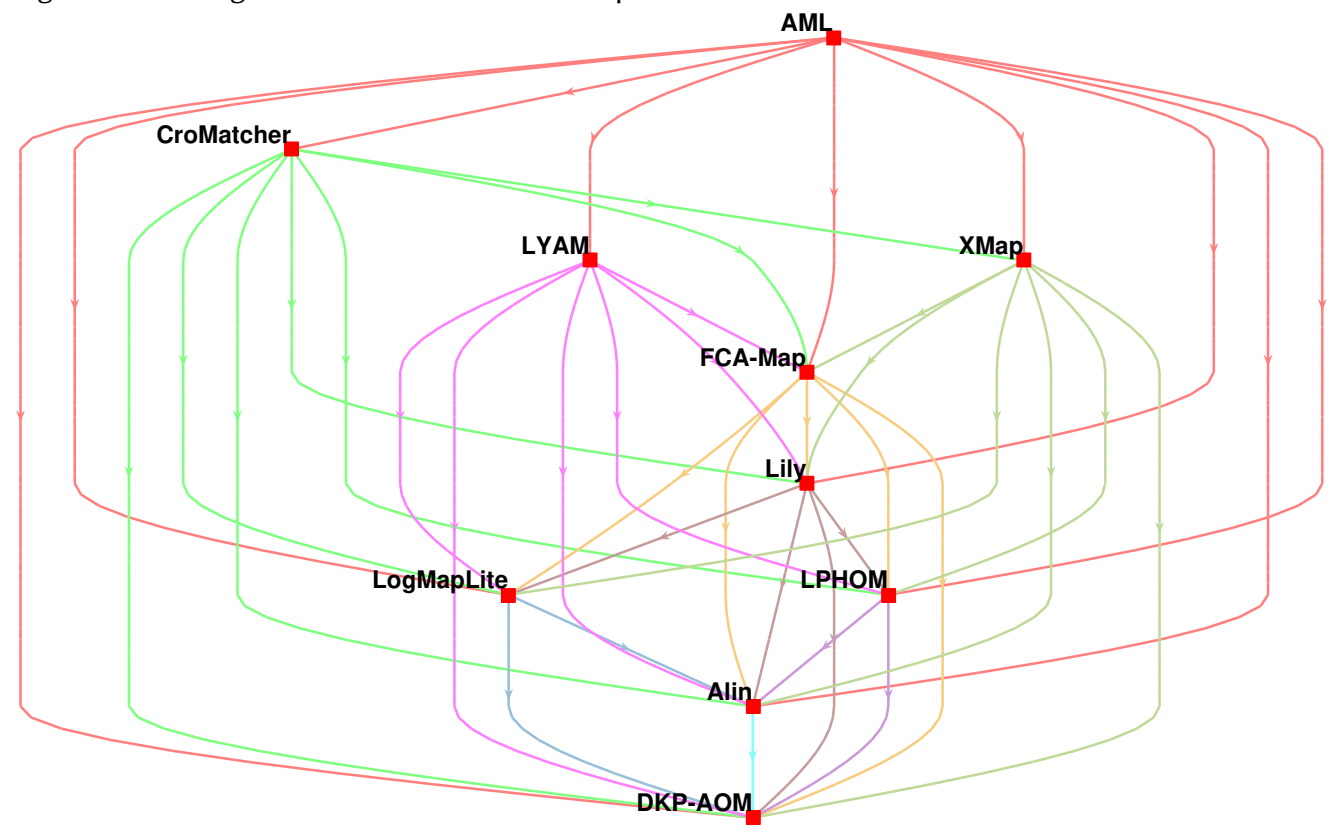

We conduct all the pairwise comparisons and we take advantage of the Nemenyi's correction and the Bergman's correction, the most conservative and most powerful ones, to control the family-wise error rate. A directed graph visualizes the outcome of the pairwise comparison. Four different directed graphs correspond to each perspective and each correction method are displayed in Figures $(1-4)$. The nodes in these graphs are the systems under study and any directed edge $A \rightarrow B$ means that $\mathrm{A}$ is significantly better than $\mathrm{B}$. If there is no such an edge, however, there is no significant difference between the corresponding systems.

First, we compare the results obtained from the Nemenyi's and Bergman's correction techniques from each perspective of the contingency table construction. Figures 1 and 2 are the directed graphs corresponds to the pairwise comparisons of alignments obtained by applying respectively the Nemenyi's and Bergmann's correction under the first perspective of contingency table construction. The results of these two correction methods are varied only in one comparison: the Bergmann's correction indicates the significant difference between CroMatcher and LYAM while the Nemenyi's correction cannot detect it. Thus, the Bergmann's correction is more powerful than the Nemenyi's method as the theory suggests.

In the second approach which considers the false positive, the Bergmann's correction indicates its power in comparison with the Nemenyi's correction. It declares the difference between FCA-Map and LYAM, and between LYAM and LogMapLite significant while the Nemenyi's correction cannot find such differences as significant.

Now, we compare the two perspectives on the contingency table construction. To do so, the Bergmann's correction method is considered due to its ability to detect more differences. Considering Fig. 2, it is readily seen that the LYAM and XMAP methods are not declared significant, but both of them are declared significant in comparison to FCA-MAP. If the false positive rate is taken into account, as in Fig. 4, FCA-MAP is replaced LYAM. To investigate such a replacement, Tables 2 and 3 must be considered. While the false positive rate is not considered, FCA-Map has 51 correct correspondences which are not in LYAM, and LYAM has 110 true 
Fig. 2. Comparison of alignment systems by the McNemar's mid-p test with the Bergmann's correction while the false positive is ignored. The edge $A \rightarrow B$ indicates that $\mathrm{A}$ outperforms $\mathrm{B}$.

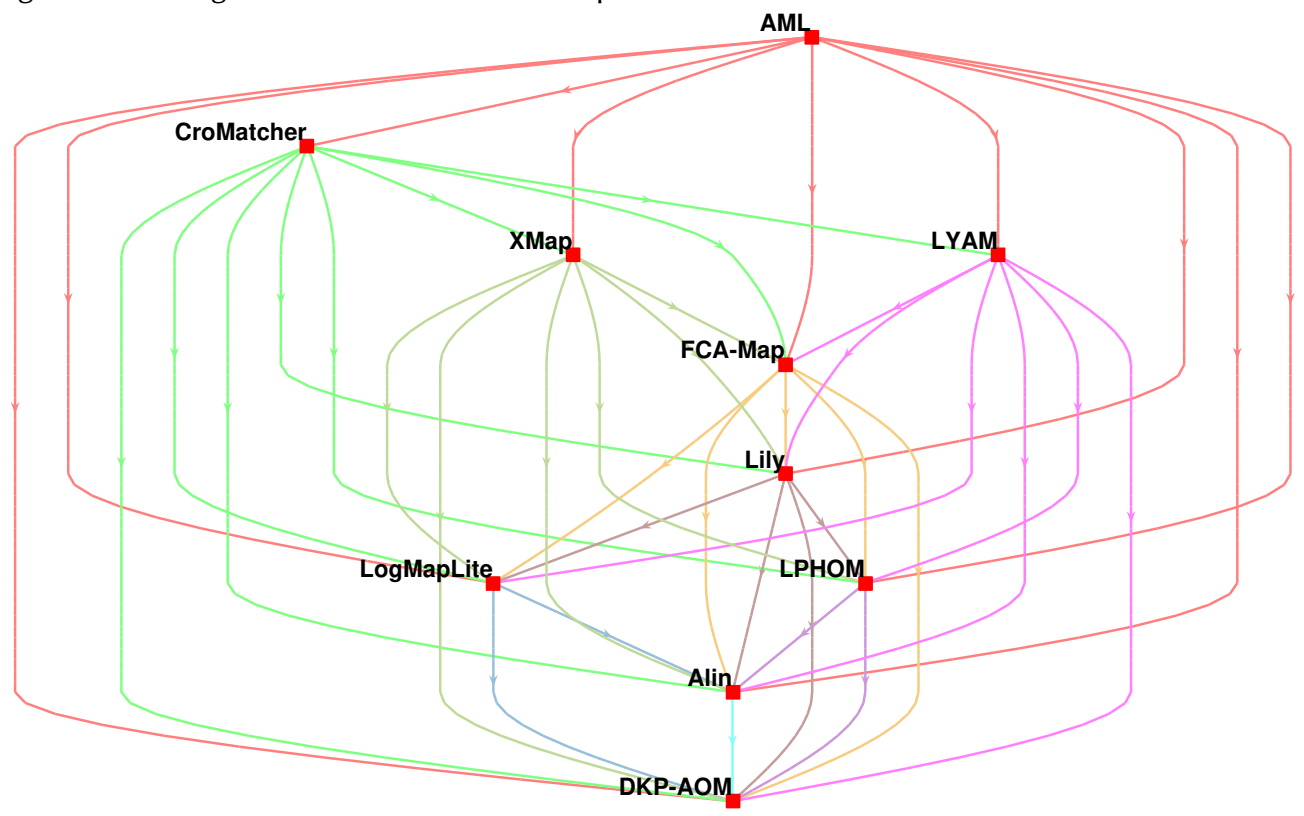

correspondences which do not exist in FCA-MAP. However, when the false positive is considered, the number of truly discovered correspondences by FCA-MAP which are not in the LYAM alignment increases to 220 while the number of truly discovered correspondences by LYAM which are not in FCA-MAP is 160. As a result, the LYAM ontology mapping is better than FCA-MAP from the first point of view, but FCA-MAP outperforms LYAM in the second approach because it has a lower false discovery rate in comparison with LYAM. The same argument is also valid for the comparison of FCA-MAP and XAMP: if the falsely discovered correspondences are not taken into account, XAMP outperforms FCA-MAP while they are declared insignificant when the false discovery error is considered as well.

Another difference between two perspectives on the contingency table construction is about the LogMapLite system. When the false discovery rate does not matter, Lily outperforms LogMapLite, which is further declared insignificant compared with LPHOM. If the false positive error is heeded, however, LogMapLite outperforms LPHOM and it is declared insignificant with Lily. This indicates that LogMapLite has a lower false discovery rate than Lily and LPHOM.

We rank the systems participated in the OAEI 2016 anatomy track in Table 4 based on the Bergmann's correction. The columns with labels IFP and CFP correspond to the contingency table construction with ignoring the false discovery (IFP) and considering (CFP) it. In this table, the systems in higher rows are ones which are significantly better than the ones in the lower rows. If two systems are not significantly different, they are placed in the same cell. It can be readily seen that AML and DKP-AOM are the best and the worst systems from two perspectives, respectively.

The results of statistical procedures are eventually compared with those of recall and F-measure. As a matter of fact, such a comparison would be of no meaning unless some circumstances would be considered. We say that two systems are not significantly different provided that their recall (or F-measure for another case) will be 
Fig. 3. Comparison of alignment systems by the McNemar's mid-p test with the Nemenyi's correction while the false positive is considered. The edge $A \rightarrow B$ indicates that A outperforms $\mathrm{B}$.

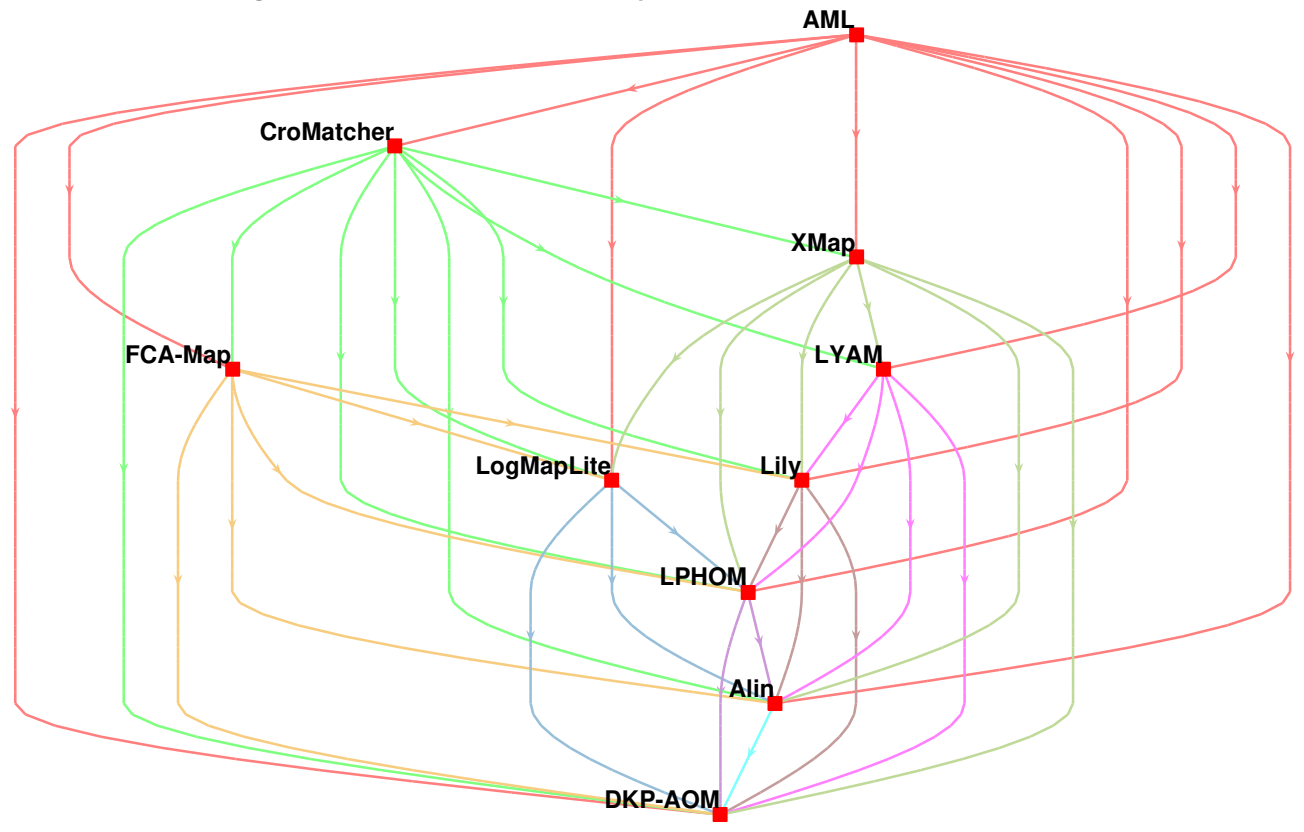

the same. Nonetheless, it must be mentioned that the comparison based on the McNemar's test is distinct from that of different performance measures. First and foremost, it does not produce any score. Second, the result of comparison might indicate that two systems are similar, the case which is not accommodated in comparison of two scores unless they are exactly the same.

First, the outcomes of our analysis from the first perspective with the Bergmann's correction (see Figure 2) is compared with the recall metric. In the OAEI 2016 anatomy track, AML and CroMatcher have the highest recall among others. At the other extreme, DKP-AOM and Alin are the systems with the least discovery. By the same token, they are the top two and bottom two systems in our analysis. One salient characteristic of the statistical analysis is the equivalence of LPHOM and LogMapLite. The recall of LogMapLite and LPHOM are 0.728 and 0.727, respectively. If the higher recall would be an indicator for superiority, then LogMapLite is declared better. However, the difference between these systems is a trifle. This triviality is reflected in the statistical analysis as they are not declared significant (there is no edge between LogMapLite and LPHOM in Figure 2). There is the same cogent argument for the comparison of XMap and LYAM.

The comparison of the second perspective is analogous to that of the F-measure. Similar to our analysis, the F-measures of AML and CroMatcher are the top systems, and those of DKP-AOM and Alin are the bottom two ones (see Figure 4).

For the final experiment, the string-based similarity measures are compared over the anatomy track. These metrics are of utmost importance, by which most of the correspondences of two given ontologies, including the ontologies of the anatomy track, could be discovered (Cheatham and Hitzler 2013). To compare such metrics over the anatomy track, we take advantage of the Shiva framework (Mathur et al. 2014) which converts the ontology mapping into an assignment problem. In this framework, the similarity between each concept from the source ontology is gauged with all the concepts of the target ontology. The similarity score between the concepts 
Fig. 4. Comparison of alignment systems by the McNemar's mid-p test with the Bergmann's correction while the false positive is considered. The edge $A \rightarrow B$ indicates that A outperforms $\mathrm{B}$.

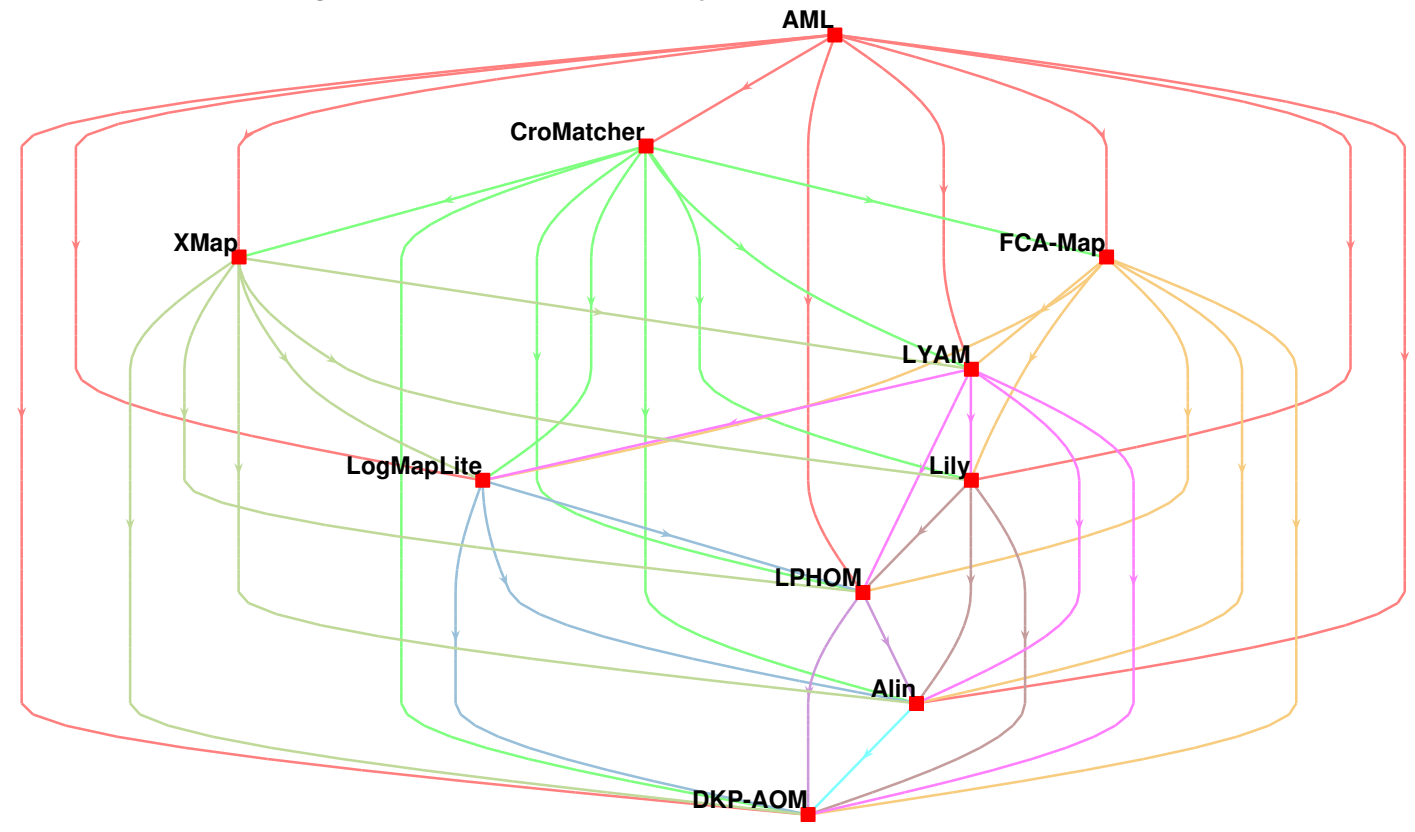

Table 4. Ranking of methods participated in the anatomy track, OAEI 2016 from two different perspectives. The first perspective is to ignore the false positive (IFP) and the second is to consider it (CFP). The position of upper rows in this table indicates that it is significantly better than the methods coming in the lower rows. Cells with two methods indicate that the methods are not declared significantly different.

\begin{tabular}{c|c|c} 
& IFP & CFP \\
\hline 1 & AML & AML \\
2 & CroMatcher & CroMatcher \\
3 & LYAM \& XAMP & FCA-MAP \& XMAP \\
4 & FCA-MAP & LYAM \\
5 & Lily & LogMapLite \& Lily \\
6 & LogMapLite \& LPHOM & LPHOM \\
7 & Alin & Alin \\
8 & DKP-AOM & DKP-AOM
\end{tabular}

of two ontologies constructs a matrix, which can be given to the Hungarian algorithm (Munkres 1957) to find the best match for each entity. We use nine string-based similarity measures to construct the matrix: Levenstein (Levenshtein 1966), N-gram (Kondrak 2005), Hamming (Euzenat et al. 2007), Jaro (Jaro 1995), JaroWinkler (Winkler 1999), SMOA (Stoilos et al. 2005), NeedlemanWunsch2 (Needleman and Wunsch 1970), Substring distance (Euzenat et al. 2007), and equivalence measure. The Hungarian method applies to the resultant matrix to find the best match for each concept. 
Table 5. The $n_{01}$ and $n_{10}$ for constructing the contingency table from the first point of view (ignoring the false positive) across the various string-based similarity measures. For the comparison of the $i^{t h}$ and $j^{t h}$ metrics, $n_{01}=(i, j)$ and $n_{10}=(j, i)$ where $(i, j)$ is the element at the $i^{t h}$ row and the $j^{\text {th }}$ column in the table.

\begin{tabular}{|c|c|c|c|c|c|c|c|c|c|}
\hline & $\underset{\text { J્| }}{\underset{J}{J}}$ & 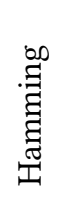 & 을 & 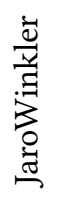 & 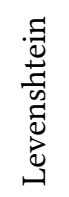 & 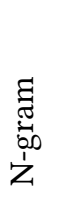 & 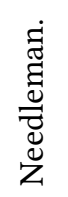 & $\sum_{\infty}^{\mathbb{S}}$ & 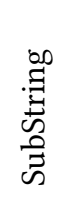 \\
\hline Equal & 0 & 0 & 2 & 2 & 0 & 0 & 0 & 71 & 0 \\
\hline Hamming & 842 & 0 & 51 & 51 & 32 & 54 & 48 & 258 & 494 \\
\hline Jaro & 888 & 95 & 0 & 0 & 42 & 59 & 60 & 252 & 532 \\
\hline JaroWinkler & 888 & 95 & 0 & 0 & 42 & 59 & 60 & 252 & 532 \\
\hline Levenshtein & 966 & 156 & 122 & 122 & 0 & 64 & 50 & 277 & 593 \\
\hline N-gram & 1041 & 253 & 214 & 214 & 139 & 0 & 174 & 290 & 636 \\
\hline Needleman. & 932 & 138 & 106 & 106 & 16 & 65 & 0 & 276 & 573 \\
\hline SMOA & 880 & 225 & 175 & 175 & 120 & 58 & 153 & 0 & 552 \\
\hline SubString & 422 & 74 & 68 & 68 & 49 & 17 & 63 & 165 & 0 \\
\hline
\end{tabular}

Fig. 5. comparison of string-based similarity measures for the anatomy track. The arrow $A \rightarrow B$ indicates that $\mathrm{A}$ outperforms B.

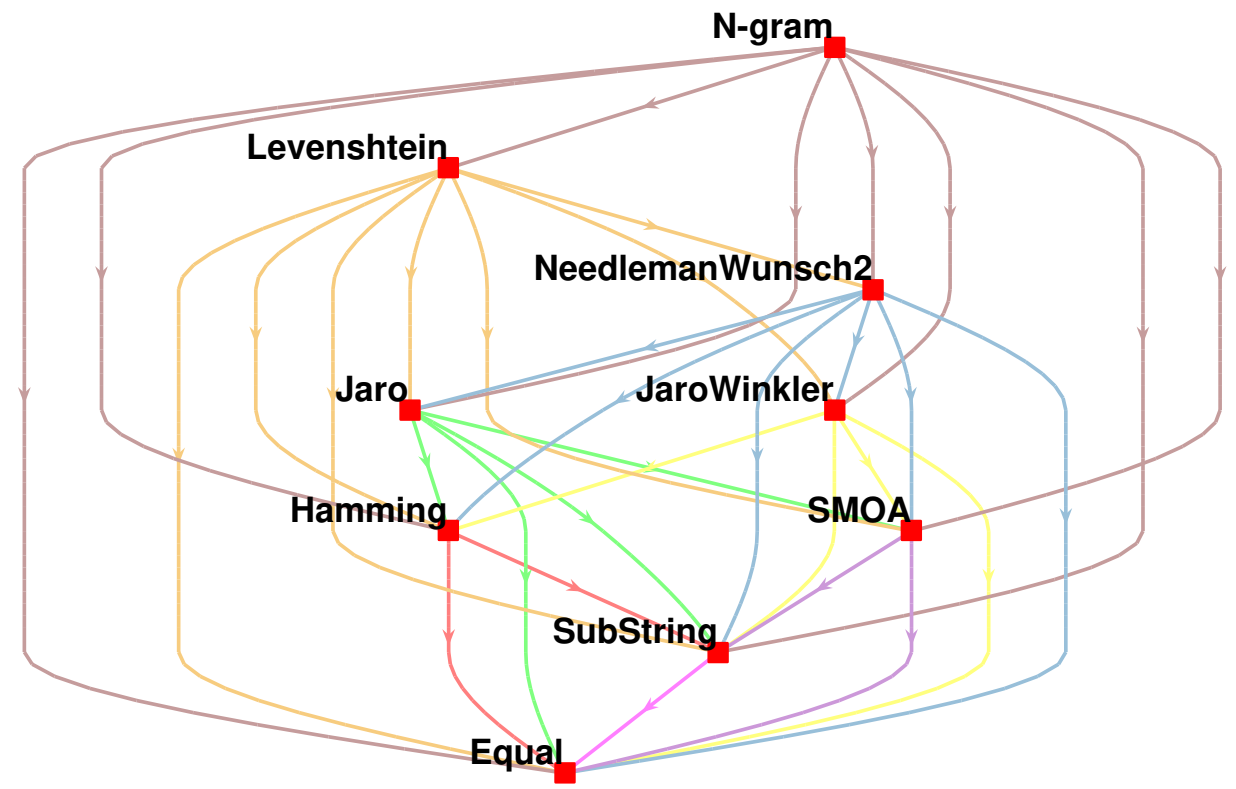

We consider the case when the false positive is not taken into account. The primary reason is that the selection of the appropriate string similarity measure can enable us to discover most of the potential correspondences (Cheatham and Hitzler 2013). If the right similarity metric is chosen, then the unreliable correspondences could be omitted by applying more strict thresholds. 
Similar to the previous ones, Table 5 tabulates $n_{01}$ and $n_{10}$ corresponding to different string-based similarity measures while the false positive is ignored. The results are visualized by a directed graph shown in Fig. 5 . From this figure, N-gram has shown the best performances and is followed by Levenstein. Further, SMOA and Hamming distances are the ones with the least retrieved correspondences but they are better than Substring and Equivalence measures as expected.

\section{CONCLUSION}

This paper proposed the utilization of the McNemar's test to compare various ontology alignment systems over one single task. The current approach for the alignment comparison is to first select a performance score and then compare two systems by obtaining their performance scores on a task with a reference alignment. In this article, the alignment produced by two systems as well as the reference alignment are given, and the outcome is if two systems are significantly different. Thus, the output is not a score, but to / not to declare the significance between two ontology matching technique. Further, the ways of preventing family-wise error rate, which is likely to happen in the comparison of multiple $(>2)$ alignment systems, are explored in minute detail. The proposed methodologies are applied to the anatomy track of ontology alignment initiative evaluation (OAEI) 2016. It is indicated that the AML and CroMatcher are the top two algorithms, and Alin and DKP-AOM are the worst alignments. For string-based similarity measures, N-gram and Levenstein outperform other methods while SMOA and Hamming distance have shown poor performances.

\section{REFERENCES}

Manel Achichi, Michelle Cheatham, Zlatan Dragisic, Jérôme Euzenat, Daniel Faria, Alfio Ferrara, Giorgos Flouris, Irini Fundulaki, Ian Harrow, Valentina Ivanova, et al. 2016. Results of the Ontology Alignment Evaluation Initiative 2016. In 11th ISWC workshop on ontology matching (OM). No commercial editor., 73-129.

Siham Amrouch, Sihem Mostefai, and Muhammad Fahad. 2016. Decision trees in automatic ontology matching. International fournal of Metadata, Semantics and Ontologies 11, 3 (2016), 180-190.

Beate Bergmann and Gerhard Hommel. 1988. Improvements of general multiple test procedures for redundant systems of hypotheses. In Multiple Hypothesenprüfung/Multiple Hypotheses Testing. Springer, 100-115.

Michelle Cheatham and Pascal Hitzler. 2013. String similarity metrics for ontology alignment. In International Semantic Web Conference. Springer, 294-309.

William Cohen, Pradeep Ravikumar, and Stephen Fienberg. 2003. A comparison of string metrics for matching names and records. In Kdd workshop on data cleaning and object consolidation, Vol. 3. 73-78.

Jomar da Silva. 2016. ALIN Results for OAEI 2016. Ontology Matching (2016), 130.

Janez Demšar. 2006. Statistical comparisons of classifiers over multiple data sets. Fournal of Machine learning research 7, Jan (2006), 1-30.

Warith Eddine Djeddi and Mohammed Tarek Khadir. 2010. XMAP: a novel structural approach for alignment of OWL-full ontologies. In Machine and Web Intelligence (ICMWI), 2010 International Conference on. IEEE, 368-373.

Olive Jean Dunn. 1961. Multiple comparisons among means. J. Amer. Statist. Assoc. 56, 293 (1961), 52-64.

Allen L Edwards. 1948. Note on the ficorrection for continuityfi in testing the significance of the difference between correlated proportions. Psychometrika 13, 3 (1948), 185-187.

Marc Ehrig and York Sure. 2004. Ontology mapping-an integrated approach. In European Semantic Web Symposium. Springer, 76-91.

Jérôme Euzenat. 2007. Semantic Precision and Recall for Ontology Alignment Evaluation.. In IfCAI. 348-353.

Jérôme Euzenat, Pavel Shvaiko, et al. 2007. Ontology matching. Vol. 18. Springer.

Morten W Fagerland, Stian Lydersen, and Petter Laake. 2013. The McNemar test for binary matched-pairs data: mid-p and asymptotic are better than exact conditional. BMC medical research methodology 13, 1 (2013), 1.

Daniel Faria, Catia Pesquita, Emanuel Santos, Matteo Palmonari, Isabel F Cruz, and Francisco M Couto. 2013. The agreementmakerlight ontology matching system. In OTM Confederated International Conferences” On the Move to Meaningful Internet Systems”. Springer, 527541.

H Finner. 1993. On a monotonicity problem in step-down multiple test procedures. F. Amer. Statist. Assoc. 88, 423 (1993), $920-923$.

Yosef Hochberg. 1988. A sharper Bonferroni procedure for multiple tests of significance. Biometrika 75, 4 (1988), 800-802.

Burt S Holland and Margaret DiPonzio Copenhaver. 1987. An improved sequentially rejective Bonferroni test procedure. Biometrics (1987), 417-423.

Sture Holm. 1979. A simple sequentially rejective multiple test procedure. Scandinavian journal of statistics (1979), 65-70. 
Sadaqat Jan, Maozhen Li, Hamed Al-Raweshidy, Alireza Mousavi, and Man Qi. 2012. Dealing with uncertain entities in ontology alignment using rough sets. IEEE Transactions on Systems, Man, and Cybernetics, Part C (Applications and Reviews) 42, 6 (2012), 1600-1612.

Matthew A Jaro. 1995. Probabilistic linkage of large public health data files. Statistics in medicine 14, 5-7 (1995), 491-498.

Ernesto Jiménez-Ruiz and Bernardo Cuenca Grau. 2011. Logmap: Logic-based and scalable ontology matching. In International Semantic Web Conference. Springer, 273-288.

Grzegorz Kondrak. 2005. N-gram similarity and distance. In International Symposium on String Processing and Information Retrieval. Springer, $115-126$.

HO Lancaster. 1961. Significance tests in discrete distributions. F. Amer. Statist. Assoc. 56, 294 (1961), 223-234.

Vladimir I Levenshtein. 1966. Binary codes capable of correcting deletions, insertions, and reversals. In Soviet physics doklady, Vol. 10. 707-710.

Iti Mathur, Nisheeth Joshi, Hemant Darbari, and Ajai Kumar. 2014. Shiva: A Framework for Graph Based Ontology Matching. arXiv preprint arXiv:1403.7465 (2014).

Quinn McNemar. 1947. Note on the sampling error of the difference between correlated proportions or percentages. Psychometrika 12, 2 (1947), 153-157.

Imen Megdiche, Olivier Teste, and Cassia Trojahn. 2016. LPHOM results for OAEI 2016. Ontology Matching (2016), 190.

George A Miller. 1995. WordNet: a lexical database for English. Commun. ACM 38, 11 (1995), 39-41.

Majid Mohammadi, Wout Hofman, and Yaohua Tan. 2018. A Comparative Study of Ontology Matching Systems via Inferential Statistics. (2018).

James Munkres. 1957. Algorithms for the assignment and transportation problems. Fournal of the society for industrial and applied mathematics 5, 1 (1957), 32-38

Miklos Nagy, Maria Vargas-Vera, and Enrico Motta. 2006. Dssim-ontology mapping with uncertainty. (2006).

Saul B Needleman and Christian D Wunsch. 1970. A general method applicable to the search for similarities in the amino acid sequence of two proteins. Fournal of molecular biology 48, 3 (1970), 443-453.

P. Nemenyi. 1963. Distribution-free multiple comparisons. Ph.D. Dissertation. Princeton University.

Dominique Ritze, Heiko Paulheim, and Kai Eckert. 2013. Evaluation measures for ontology matchers in supervised matching scenarios. In International Semantic Web Conference. Springer, 392-407.

Juliet Popper Shaffer. 1986. Modified sequentially rejective multiple test procedures. F. Amer. Statist. Assoc. 81, 395 (1986), $826-831$.

Juliet Popper Shaffer. 1995. Multiple hypothesis testing. Annual review of psychology 46, 1 (1995), 561-584.

David J Sheskin. 2003. Handbook of parametric and nonparametric statistical procedures. crc Press.

Giorgos Stoilos, Giorgos Stamou, and Stefanos Kollias. 2005. A string metric for ontology alignment. In International Semantic Web Conference. Springer, 624-637.

Samy Suissa and Jonathan J Shuster. 1991. The 2 x 2 matched-pairs trial: Exact unconditional design and analysis. Biometrics (1991), 361-372.

Peng Wang and Baowen Xu. 2008. Lily: Ontology alignment results for oaei 2008. In Proceedings of the 3rd International Conference on Ontology Matching-Volume 431. CEUR-WS. org, 167-175.

William E Winkler. 1999. The state of record linkage and current research problems. In Statistical Research Division, US Census Bureau. Citeseer.

David H Wolpert. 1996. The lack of a priori distinctions between learning algorithms. Neural computation 8, 7 (1996), 1341-1390.

David H Wolpert. 2012. What the no free lunch theorems really mean; how to improve search algorithms. In Santa fe Institute Working Paper. 12.

David H Wolpert and William G Macready. 1997. No free lunch theorems for optimization. IEEE transactions on evolutionary computation 1, 1 (1997), 67-82.

Xingsi Xue and Yuping Wang. 2015. Ontology alignment based on instance using NSGA-II. fournal of Information Science 41, 1 (2015), 58-70.

Mengyi Zhao and Songmao Zhang. 2016. FCA-Map Results for OAEI 2016. Ontology Matching (2016), 172.

Received March 2017; revised January 2018; accepted March 2018 


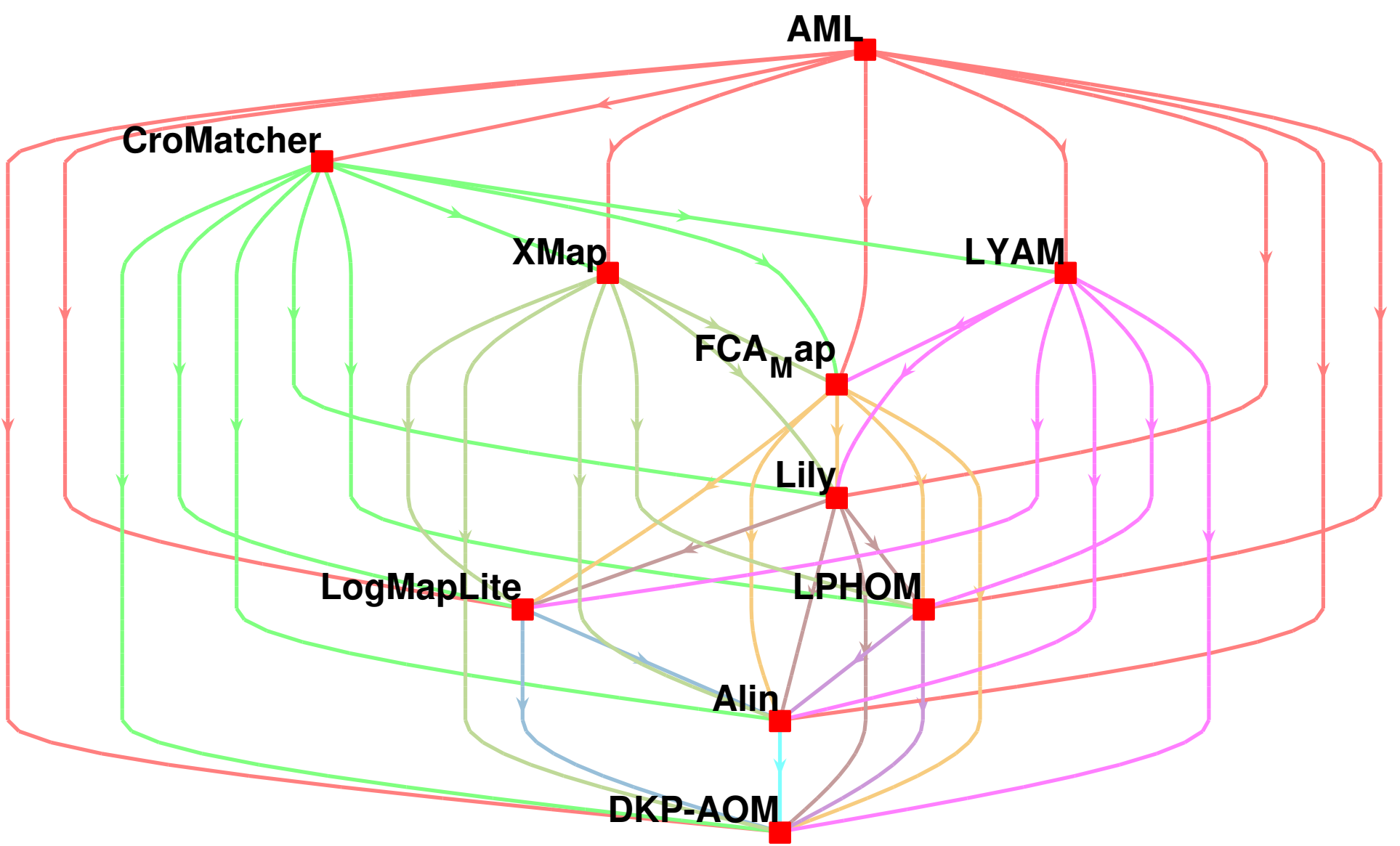

